\title{
Minimal extension of the standard model scalar sector
}

\author{
Donal O’Connell, ${ }^{1}$ Michael J. Ramsey-Musolf, ${ }^{1,2}$ and Mark B. Wise ${ }^{1}$ \\ ${ }^{1}$ California Institute of Technology, Pasadena, California 91125, USA \\ ${ }^{2}$ University of Wisconsin-Madison, Madison, Wisconsin 53706, USA
}

(Received 21 November 2006; published 13 February 2007)

\begin{abstract}
The minimal extension of the scalar sector of the standard model contains an additional real scalar field with no gauge quantum numbers. Such a field does not couple to the quarks and leptons directly but rather through its mixing with the standard model Higgs field. We examine the phenomenology of this model focusing on the region of parameter space where the new scalar particle is significantly lighter than the usual Higgs scalar and has small mixing with it. In this region of parameter space most of the properties of the additional scalar particle are independent of the details of the scalar potential. Furthermore the properties of the scalar that is mostly the standard model Higgs can be drastically modified since its dominant branching ratio may be to a pair of the new lighter scalars.
\end{abstract}

DOI: 10.1103/PhysRevD.75.037701

PACS numbers: $14.80 . \mathrm{Cp}$

\section{INTRODUCTION}

Many of the extensions of the standard model that are testable at the LHC have been motivated by the hierarchy puzzle. However, the small value of the cosmological constant requires a fine tuning that in many ways is quite similar to the fine tuning needed to keep the Higgs scalar light. Most extensions of the standard model that are constructed to solve the hierarchy puzzle still require a fine tuning to keep the cosmological constant small. It is possible that we are not looking at this issue correctly and that stability of the radiatively corrected scalar potential should not be the motivation for extensions of the standard model.

The scalar sector of the standard model has not been tested directly by experiment, so it is certainly worth examining models with simple extensions of this sector and exploring their phenomenology in some detail. There does not seem to be a compelling motivation for a scalar sector that consists of just a single Higgs doublet. However, if one does not adopt additional symmetry principles $[1,2]$ then adding more doublets typically gives unacceptably large tree level flavor changing neutral currents. Furthermore scalar fields with nontrivial $S U(2) \times U(1)$ quantum numbers that are different from those of the standard model Higgs doublet must have small vacuum expectation values to preserve the standard model value of the $\rho$ parameter. The phenomenology of the standard model Higgs scalar, models with multiple Higgs doublets, and of supersymmetric extensions of the standard model has been studied extensively, and Ref. [3] contains some excellent reviews.

The simplest extension of the scalar sector of the minimal standard model is to add a single real scalar $S$ that is a gauge singlet. This does not have to be a fundamental degree of freedom; the Higgs doublet and this scalar might be the only light remnants of a more complicated scalar sector that manifests itself at scales that are too high to be directly probed by the next generation of accelerator experiments. In this paper we examine the phenomenology of this extension of the standard model. Extensions of the minimal standard model with one or more singlets $S$ have been studied before in the literature. Many of the models impose a $S \rightarrow-S$ symmetry, so that the singlet can be a dark matter candidate. Other works (for example, see Ref. [4]) either do not impose a $S \rightarrow-S$ symmetry or break that symmetry, but have some differences with the model we present here (e.g., some possible couplings in the scalar potential are missing, or the lighter scalar is taken to be massless, etc.) In any case, it seems worth reexamining the phenomenology of this model since we are approaching the LHC era. Our work was inspired by Ref. [5] where an additional scalar superfield was added to the minimal supersymmetric standard model to solve the $\mu$ problem, and some of our conclusions are similar to theirs. ${ }^{1}$

In the minimal standard model the scalar potential for the Higgs doublet $H$ contains only two parameters which can be eliminated in favor of the Higgs particle mass and the vacuum expectation value that breaks $S U(2) \times U(1)$ gauge symmetry. When the singlet scalar $S$ is added the number of parameters of the scalar potential swells to seven. However for most of the phenomenology only a few parameters are relevant, and a very simple picture emerges. The singlet scalar $S$ and the Higgs scalar $h$ mix, and both of the resulting physical particles have couplings to quarks, leptons and to gauge bosons that are proportional to those of the standard model Higgs particle. In addition to decays to the standard model fermions and gauge bosons, the heavier of these two scalar particles may decay to a pair of the lighter ones.

In this brief report we focus on the region of parameter space where the lighter of the two scalar particles is mostly singlet and has a small enough mass so that it can be pairproduced in decays of the heavier (mostly) Higgs scalar. This will be the most interesting case for LHC physics. The only new parameters (beyond those in the standard model)

\footnotetext{
${ }^{1}$ See also Ref. [6].
} 
that are needed to characterize most of the phenomenology of this model are the $h-S$ mixing angle, the mass of the new light scalar particle, and the branching ratio for the decay of the heavier Higgs scalar to a pair of the lighter ones.

\section{SCALAR POTENTIAL} is

The Lagrange density for the scalar sector of this model

$$
\mathcal{L}=\left(D_{\mu} H\right)^{\dagger} D^{\mu} H+\frac{1}{2} \partial_{\mu} S \partial^{\mu} S-V(H, S),
$$

where $H$ denotes the complex Higgs doublet and $S$ the real scalar. Without loss of generality, we shift the field $S$ so it has no vacuum expectation value. Then the potential is given by

$$
\begin{aligned}
V(H, S)= & \frac{m^{2}}{2} H^{\dagger} H+\frac{\lambda}{4}\left(H^{\dagger} H\right)^{2}+\frac{\delta_{1}}{2} H^{\dagger} H S \\
& +\frac{\delta_{2}}{2} H^{\dagger} H S^{2}+\left(\frac{\delta_{1} m^{2}}{2 \lambda}\right) S+\frac{\kappa_{2}}{2} S^{2} \\
& +\frac{\kappa_{3}}{3} S^{3}+\frac{\kappa_{4}}{4} S^{4} .
\end{aligned}
$$

Note that there is no additional $C P$ violation that comes from the scalar potential.

In unitary gauge the charged component of the Higgs doublet $H$ becomes the longitudinal components of the charged $W$-bosons and the imaginary part of the neutral component becomes the longitudinal component of the $Z$-boson. The neutral component is written as

$$
H^{0}=\frac{v+h}{\sqrt{2}}, \quad v=\sqrt{\frac{-2 m^{2}}{\lambda}} .
$$

The mass terms in the scalar potential become

$$
V_{\text {mass }}=\frac{1}{2}\left(\mu_{h}^{2} h^{2}+\mu_{S}^{2} S^{2}+\mu_{h S}^{2} h S\right),
$$

where

$$
\begin{gathered}
\mu_{h}^{2}=-m^{2}=\lambda v^{2} / 2 \quad \mu_{S}^{2}=\kappa_{2}+\delta_{2} v^{2} / 2 \\
\mu_{h S}^{2}=\delta_{1} v .
\end{gathered}
$$

The mass eigenstate fields $h_{+}$and $h_{-}$are linear combinations of the Higgs scalar field $h$ and the singlet scalar field $S$. Explicitly, for the lighter field $h_{-}$

$$
h_{-}=\cos \theta S-\sin \theta h \text {, }
$$

where [4]

$$
\tan \theta=\frac{x}{1+\sqrt{1+x^{2}}}, \quad x=\frac{\mu_{h S}^{2}}{\mu_{h}^{2}-\mu_{S}^{2}} .
$$

The terms in the scalar potential that break the discrete $S \rightarrow-S$ symmetry are proportional to the couplings $\delta_{1}$ and $\kappa_{3}$ so it is natural for those scalar couplings to be small. The parameter $\delta_{1}$ controls the mixing of the two scalar states. As we will see, experimental constraints force the mixing angle $\theta$ to be small. We assume the heavier state is mostly the Higgs scalar so $\mu_{h}^{2}>\mu_{S}^{2}$. The masses of the two scalars are

$$
m_{ \pm}^{2}=\left(\frac{\mu_{h}^{2}+\mu_{S}^{2}}{2}\right) \pm\left(\frac{\mu_{h}^{2}-\mu_{S}^{2}}{2}\right) \sqrt{1+x^{2}} .
$$

\section{PHENOMENOLOGY}

The lighter scalar state decays to standard model particles and its couplings to them are proportional to the standard model Higgs couplings with constant of proportionality $\sin \theta$. Consequently, the lighter of the two states has branching ratios equal to those of the standard model Higgs (if it had mass $m_{-}$) and its production rates $\operatorname{are~}^{2} \sin ^{2} \theta$ times the production rates for a standard model Higgs (if it had mass $m_{-}$). Since $\sin \theta$ can be much smaller than unity, $m_{-}$can be much smaller than the mass of the standard model Higgs, which is restricted by the LEP bound to be heavier than $114 \mathrm{GeV}$.

If $m_{+}<2 m_{-}$then the heavier state has branching ratios to standard model particles and production rates approximately equal to those of the standard model Higgs scalar (recall we are working in the limit of small mixing). However if $m_{+}>2 m_{-}$then the decay channel $h_{+} \rightarrow$ $h_{-} h_{-}$is available with partial decay width

$$
\Gamma\left(h_{+} \rightarrow h_{-} h_{-}\right)=\frac{\delta_{2}^{2} v^{2}}{32 \pi m_{+}} \sqrt{1-4 m_{-}^{2} / m_{+}^{2}} .
$$

For a $h_{+}$that has mass below $140 \mathrm{GeV}$ its dominant decay mode to standard model particles is to a bottom-antibottom pair and so in this mass range

$$
\frac{\Gamma\left(h_{+} \rightarrow h_{-} h_{-}\right)}{\Gamma^{\text {S.M. }}(h)} \simeq \frac{\delta_{2}^{2} v^{4}}{6 m_{+}^{2} m_{b}^{2}} \sqrt{1-4 m_{-}^{2} / m_{+}^{2}},
$$

where $\Gamma^{\text {S.M. }}(h)$ denotes the decay width of the standard model Higgs. Conventional branching ratios of the heavier scalar $h_{+}$are reduced from those of the standard model Higgs by a factor $f$ which is equal to

$$
f=\frac{1}{1+\Gamma\left(h_{+} \rightarrow h_{-} h_{-}\right) / \Gamma^{\mathrm{S} . \mathrm{M} .}(h)}=1-\operatorname{Br}\left(h_{+} \rightarrow h_{-} h_{-}\right) .
$$

\section{A. Very light $\boldsymbol{h}_{-}$}

If $m_{-}$is much smaller than the weak scale it seems most natural to take $\left|\delta_{2}\right|$ to be of order $2 m_{-}^{2} / v^{2}$ or smaller, since if it is much larger than this a strong cancellation between the two terms contributing to $\mu_{S}^{2}$ in Eq. (5) is necessary. Suppose $m_{-}$is less than the $b$-quark mass, $m_{b}$. With $\left|\delta_{2}\right| \sim$ $2 m_{-}^{2} / v^{2}$ we find that in this region of parameter space $f$ is very close to unity since $\Gamma\left(h_{+} \rightarrow h_{-} h_{-}\right) / \Gamma^{\text {S.M. }}(h) \sim$ $\left(m_{-} / m_{b}\right)^{2}\left(m_{-} / m_{+}\right)^{2} \ll 1$. The heavier scalar state will behave much like the standard model Higgs particle. 
The properties of a very light $h_{-}$are severely constrained by present experimental data. Consider, for example, the case where $m_{-}=500 \mathrm{MeV}$. Then the branching ratio of the $h_{+}$to two $h_{-}$'s is very small and the $h_{+}$is indistinguishable from the standard model Higgs particle. The dominant decay modes of the $h_{-}$are to two pions and to $\mu^{+} \mu^{-}$with partial decay rates, ${ }^{2}$

$$
\begin{aligned}
\Gamma\left(h_{-} \rightarrow \pi^{+} \pi^{-}\right) & =2 \Gamma\left(h_{-} \rightarrow \pi^{0} \pi^{0}\right) \\
& =\frac{\sin ^{2} \theta m_{-}^{3}}{324 v^{2} \pi}\left[1+\frac{11}{2}\left(\frac{m_{\pi}^{2}}{m_{-}^{2}}\right)\right]^{2} \sqrt{1-\frac{4 m_{\pi}^{2}}{m_{-}^{2}},}
\end{aligned}
$$

and

$$
\Gamma\left(h_{-} \rightarrow \mu^{+} \mu^{-}\right)=\frac{\sin ^{2} \theta m_{-} m_{\mu}^{2}}{8 v^{2} \pi}\left[1-\frac{4 m_{\mu}^{2}}{m_{-}^{2}}\right]^{3 / 2} .
$$

These imply that $\operatorname{Br}\left(h_{-} \rightarrow \mu^{+} \mu^{-}\right) \simeq 34 \%$ and that the lifetime of the $h_{-}$is $\tau_{h_{-}} \simeq\left(9 / \sin ^{2} \theta\right) \times 10^{-17} \mathrm{sec}$. A strong constraint on the mixing angle $\theta$ comes from the decay $B \rightarrow h_{-} X$ which has branching ratio $\operatorname{Br}(B \rightarrow$ $\left.h_{-} X\right) \simeq 8 \sin ^{2} \theta$. The experimental limit $\operatorname{Br}(B \rightarrow$ $\left.\mu^{+} \mu^{-} X\right)<3.2 \times 10^{-4}$ [8] implies that $\sin ^{2} \theta<1 \times$ $10^{-4}$. Hence the lifetime of the $h_{-}$is at least $8 \times$ $10^{-13} \mathrm{sec}$ (i.e., about the $B$ meson lifetime). It may be possible to derive stronger constraints on the mixing angle from exclusive decays of the $B$ meson.

The $h_{-}$can be produced directly in $Z$ decays. Single $h_{-}$ production is suppressed by the small mixing angle, $\operatorname{Br}\left(Z \rightarrow h_{-} \bar{f} f\right) / \operatorname{Br}(Z \rightarrow \bar{f} f) \simeq 10^{-2} \sin ^{2} \theta<1 \times 10^{-6}$, where $f$ denotes any of the light standard model fermions. The $h_{-}$can also be pair-produced through a virtual $h_{+}$, with a rate that is not suppressed by the small mixing angle $\theta$, via the process $Z \rightarrow h_{+}^{*} \bar{f} f \rightarrow h_{-} h_{-} \bar{f} f$. We find that this rate is negligible ${ }^{3}$ when $\left|\delta_{2}\right| \sim 2 m_{-}^{2} / v^{2}$.

\section{B. $5 \mathrm{GeV}<\boldsymbol{m}_{-}<50 \mathrm{GeV}$}

This mass range is interesting because the decays of the heavier scalar, which is mostly the standard model Higgs, can be quite different from what the minimal standard model predicts. In this mass range the $h_{-}$is light enough that the decay $h_{+} \rightarrow h_{-} h_{-}$is kinematically allowed. In addition the $h_{-}$is heavy enough so that values of $\delta_{2}$ that give this decay a significant branching ratio do not require a delicate cancellation between the two terms contributing to $\mu_{S}^{2}$ in Eq. (5). At the lower end of this mass range we would not expect the dominant decay of the $h_{+}$to be to two $h_{-}$'s; however, with no awkward cancellation between the

\footnotetext{
${ }^{2}$ The rate to two pions is calculated at leading order in chiral perturbation theory [7]. With $m_{-}=500 \mathrm{MeV}$ one can expect sizeable corrections from, for example, $\pi \pi$ final state interactions. These are expected to increase the decay rate to two pions.

${ }^{3}$ For $\kappa_{2}=0, m_{+}=120 \mathrm{GeV}$ and $m_{-}=5 \mathrm{GeV}$, we find that $\operatorname{Br}\left(Z \rightarrow h_{+}^{*} \bar{f} f \rightarrow h_{-} h_{-} \bar{f} f\right) / \operatorname{Br}(Z \rightarrow \bar{f} f)=5.4 \times 10^{-13}$. The rate is even smaller for smaller values of $m_{-}$.
}

two terms contributing to $\mu_{S}^{2}$, the $h_{+}$could easily decay about the same amount of time to two $h_{-}$'s as it does to two photons. At the upper end of the mass range it is quite reasonable to have the $h_{+}$decaying mostly to two $h_{-}$'s.

In Fig. 1 we plot the suppression factor $f=$ $1-\operatorname{Br}\left(h_{+} \rightarrow h_{-} h_{-}\right)=\operatorname{Br}\left(h_{+} \rightarrow \gamma \gamma\right) / \mathrm{Br}^{\text {S.M. }}(h \rightarrow \gamma \gamma)$, where $\mathrm{Br}^{\mathrm{S} . \mathrm{M}}(h \rightarrow \gamma \gamma)$ is the standard model branching fraction, assuming that the parameter $\kappa_{2}=$ $-\delta_{2} v^{2} / 4,0,+\delta_{2} v^{2} / 4,+\delta_{2} v^{2}$ and that the mixing angle $\theta$ is very small. Notice that as one approaches the upper range of the mass range we are considering, decays of the $h_{+}$can be dominated by the final state $h_{-} h_{-}$, and consequently the branching ratio to the two photon mode is suppressed compared to what it is in the standard model. The dependence of $f$ on the mass of the $h_{-}$arises because the same coupling in the Lagrangian that gives rise to this branching ratio also contributes to the $h_{-}$mass. If $\kappa_{2}$ is larger than $\delta_{2} v^{2}$, then $f$ will be close to unity throughout the whole range we consider.

Since the $h_{+}$may decay dominantly to a pair of the lighter $h_{-}$scalars it is useful to understand the ensuing $h_{-}$ decays. We plot the branching ratios of the $h_{-}$in Fig. 2. In the upper end of the mass range we are considering, the $h_{-}$ decays mostly to a bottom-antibottom pair but it also has substantial branching fractions to $c \bar{c}$ and to $\tau^{+} \tau^{-}$. Considerable attention has been given to a different class of models where the heavier Higgs scalar decays mostly to a singlet scalar that consequently decays to neutrinos [9].

In Ref. [10] it was noted that new physics at the $\mathrm{TeV}$ scale can easily give rise to a large reduction (or enhancement) in the dominant gluon fusion Higgs scalar production rate, and hence the final two photon signal. However, it is very unlikely that such physics could alter the associated production rate of the Higgs since it arises from the tree coupling to the massive weak bosons. The situation is different here. All the standard model decays of the heavier Higgs-like scalar $h_{+}$are reduced by the same factor $f$ independent of its production mechanism.

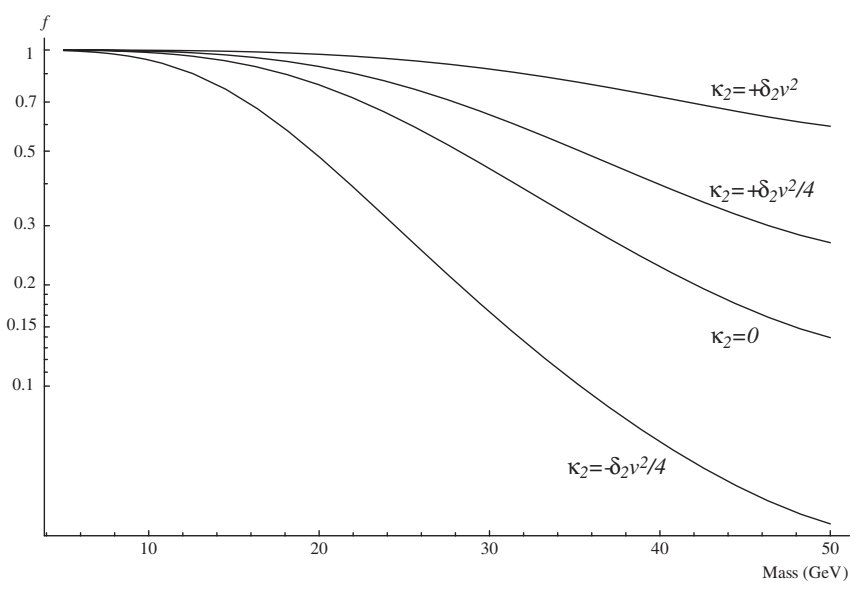

FIG. 1. The suppression factor $f$ discussed in the text, plotted as a function of the $h_{-}$mass. 


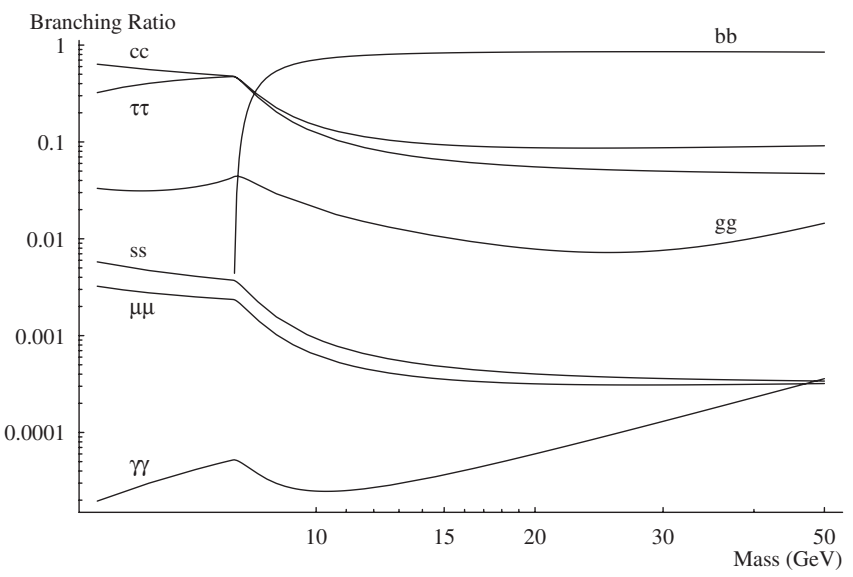

FIG. 2. The branching ratios of the light $h_{-}$scalar particle, plotted as a function of its mass.

The production of $h_{-}$scalars from gluon fusion is suppressed from the production rate for a standard model Higgs of the same mass by $\sin ^{2} \theta$. Since the $h_{-}$was not observed at LEP there is a mass dependent limit on $\sin ^{2} \theta$ from LEP data [11] (Very roughly, $\sin ^{2} \theta<2 \times 10^{-2}$ over the mass range we are considering.). However, the $h_{-}$ production rate via gluon fusion at the Tevatron and LHC increases rapidly as its mass decreases. For example, using leading order CTEQ5 parton distributions [12] an $h_{-}$of mass $10 \mathrm{GeV}$ has a production rate roughly 100 times greater than one with a mass of $120 \mathrm{GeV}$ at the LHC and 1000 times greater at the Tevatron. Even with a small value for $\sin ^{2} \theta$ the $h_{-}$may be directly observable at the LHC.

\section{CONCLUDING REMARKS}

The literature on extensions of the minimal standard model with a more complicated scalar sector is vast. Here, we have considered the simplest alternative possibility, in which a single gauge-singlet scalar is added. It mixes with, and couples to, the standard model Higgs. We concentrate on the region of allowed singlet scalar masses where it is light enough to be pair-produced in Higgs decay and, hence, suppress the Higgs "golden mode" branching ratio to two photons. We found that this suppression is unlikely to be significant if the new scalar is very light but can easily be large if the (mostly) singlet scalar is heavier than about $10 \mathrm{GeV}$. Under this scenario, there is a scalar that has a mass below the LEP Higgs mass lower bound with decay branching ratios that are identical to those of a standard model Higgs of the same mass. However its production rate is suppressed by a small mixing angle. Potential signatures of such light scalars include the observation of Higgs decay products with invariant mass well below $114 \mathrm{GeV}$ or unusual final states in Higgs decay such as two $b$-jets and a $\tau^{+} \tau^{-}$(or $\left.\mu^{+} \mu^{-}\right)$pair.
[1] S. L. Glashow and S. Weinberg, Phys. Rev. D 15, 1958 (1977).

[2] R. S. Chivukula and H. Georgi, Phys. Lett. B 188, 99 (1987); G. D’Ambrosio, G. F. Giudice, G. Isidori, and A. Strumia, Nucl. Phys. B645, 155 (2002).

[3] R. N. Cahn, Rep. Prog. Phys. 52, 389 (1989); J. F. Gunion, H. E. Haber, G. Kane, and S. Dawson, The Higgs Hunter's Guide, Frontiers in Physics (Perseus Publishing, Cambridge, 1990); L. Reina, hep-ph/0512377.

[4] N. V. Krasnikov, Phys. Lett. B 291, 89 (1992); Mod. Phys. Lett. A 13, 893 (1998); A. Datta and A. Raychaudhuri, Phys. Rev. D 57, 2940 (1998); D. G. Cerdeno, A. Dedes, and T.E. J. Underwood, J. High Energy Phys. 09 (2006) 067.

[5] J. Ellis, J. F. Gunion, H. E. Haber, L. Roszkowski, and F. Zwirner, Phys. Rev. D 39, 844 (1989); U. Ellwanger, J. F. Gunion, and C. Hugonie, hep-ph/0111179; U. Ellwanger, J. F. Gunion, C. Hugonie, and S. Moretti, hep-ph/0401228; R. Dermisek and J. F. Gunion, Phys. Rev. Lett. 95, 041801
(2005); R. Dermisek and J. F. Gunion, Phys. Rev. D 73, 111701 (2006).

[6] V. Barger, P. Langacker, H. S. Lee, and G. Shaughnessy, Phys. Rev. D 73, 115010 (2006).

[7] M. Voloshin and V. Zakharov, Phys. Rev. Lett. 45, 688 (1980); M. Voloshin, Sov. J. Nucl. Phys. 44, 478 (1986); R. S. Chivukula, A. G. Cohen, H. Georgi, B. Grinstein, and A. V. Manohar, Ann. Phys. (N.Y.) 192, 93 (1989).

[8] W. M. Yao et al. (Particle Data Group), J. Phys. G 33, 1 (2006).

[9] R. E. Shrock and M. Suzuki, Phys. Lett. B 110, 250 (1982); L. F. Li, Y. Liu, and L. Wolfenstein, Phys. Lett. B 159, 45 (1985); A. S. Joshipura and J. W. Valle, Nucl. Phys. B397, 105 (1993).

[10] A. V. Manohar and M. B. Wise, Phys. Lett. B 636, 107 (2006); Phys. Rev. D 74, 035009 (2006).

[11] R. Barate et al. (LEP Working Group for Higgs boson searches), Phys. Lett. B 565, 61 (2003).

[12] CTEQ Collaboration, http://www.phys.psu.edu/ cteq/. 